\title{
Collective chemotaxis of active nematic droplets
}

\author{
Rian Hughes and Julia M Yeomans \\ Rudolf Peierls Centre for Theoretical Physics, University of Oxford, Oxford OX1 3PU, UK
}

(Dated: August 19, 2020)

\begin{abstract}
Collective chemotaxis plays a key role in the navigation of cell clusters in e.g. embryogenesis and cancer metastasis. Using the active nematic continuum equations, coupled to a chemical field that regulates activity, we demonstrate and explain a physical mechanism that results in collective chemotaxis. The activity naturally leads to cell polarisation at the cluster interface which induces outwards flows. The chemical gradient then breaks the symmetry of the flow field, leading to a net motion. The velocity is independent of the cluster size in agreement with experiment.
\end{abstract}

Introduction: Chemotaxis denotes the movement of a motile cell in the direction of an increasing or decreasing chemical gradient. It is one of the main mechanisms that provides cells with a sense of direction in a complex environment. There is now considerable evidence that aggregates of cells can also chemotax, a phenomenon termed collective chemotaxis [1-5]. For example, lymphoid, endothelial, glial and neural crest cells have all been shown to move collectively along chemical concentration gradients [6-9]. Collective chemotaxis plays a vital role in biological processes such as embryogenesis and cancer metastasis [1, 10].

Surprisingly, the behaviour of chemotaxing clusters can be distinct from single-cell chemotaxis. Experiments have demonstrated that clusters of cells can chemotax as a collective, regardless of whether the constituent cells are capable [7, 8] or incapable [1] of responding to a chemical gradient. Moreover there is strong evidence for the importance of cellcell junctions $[1,4]$. This suggests that cluster chemotaxis is an emergent phenomenon, and cell and agent-based numerical models have been used to suggest generic mechanisms. Examples include flocking models, coupling chemokine gradients to contact inhibition of locomotion and considering chemical signalling between cells [11-14].

Recently there has been widespread interest in interpreting the dynamics of cell colonies in terms of the properties of active nematics $[15,16]$. Examples include motile topological defects in both colonies of spindle-shaped cells and in epithelial sheets, strong vorticity correlations in cell monoloyers, and spontaneous unidirectional flow in confinement [17-20]. Building upon these ideas we couple the hydrodynamic active nematic equations of motion, which capture the physics of a confluent layer of self-propelling cells at the coarse-grained level, to a dynamically evolving chemical field. Allowing the cell activity to depend on the local concentration of the chemoattractant, we show that cell clusters move up a chemical gradient. We argue that the mechanism driving the chemotactic response is the activity-driven alignment of cells on the cluster boundary, and show that the model reproduces experimental results.

Model: Our aim is to model the dynamical behaviour of a cell colony in the presence of a diffusing chemical species that controls the local activity and is adsorbed by the cells. We use a continuum approach, and describe the cell colony as a two-dimensional active nematic [18]. The governing hydro- dynamic equations couple four variables; $\mathbf{Q}$ and $\mathbf{u}$ which describe the local nematic order and velocity of the cells, the cell concentration, $\phi$, which allows the position of the colony to be tracked, and $C$, the concentration field of the chemoattractant.

In two dimensions the nematic order parameter tensor $\mathbf{Q}$ is related to the coarse-grained average of the local director, $\mathbf{n}$, by $\mathbf{Q}=S(\mathbf{n n}-\mathbf{I} / 2)$ where $S$ is the magnitude of the nematic order. The dynamics of $\mathbf{Q}$ is governed by the equation,

$$
\left(\partial_{t}+\boldsymbol{u} \cdot \boldsymbol{\nabla}\right) \mathbf{Q}-\mathbf{S}=\Gamma_{Q} \mathbf{H},
$$

where $\mathbf{S}=\lambda \mathbf{E}-(\mathbf{\Omega} \cdot \mathbf{Q}-\mathbf{Q} \cdot \mathbf{\Omega})$ is a generalised advection term characterising the response of the nematic tensor to velocity gradients. Here, $\mathbf{E}=\left(\boldsymbol{\nabla} \boldsymbol{u}+\boldsymbol{\nabla} \boldsymbol{u}^{T}\right) / 2$ is the strain rate tensor, $\boldsymbol{\Omega}=\left(\boldsymbol{\nabla} \boldsymbol{u}^{T}-\boldsymbol{\nabla} \boldsymbol{u}\right) / 2$ the vorticity tensor, and $\lambda$ is the alignment parameter. $\Gamma_{Q}$ is a rotational diffusivity and the molecular field $\mathbf{H}=-\frac{\delta \mathscr{\mathcal { F }}}{\delta \mathbf{Q}}+\nabla \cdot \frac{\delta \mathcal{F}}{\delta \nabla \mathbf{Q}}$, models the relaxation of the orientational order to the minimum of the free energy density,

$$
\mathcal{F}=A_{\phi}(\phi)^{2}(1-\phi)^{2}+K_{\phi}(\nabla \phi)^{2}+B\left(\phi-S^{2}\right)^{2}+K_{\mathbf{Q}}(\nabla \mathbf{Q})^{2} .
$$

The variable $\phi$ distinguishes the region occupied by the active nematic $(\phi=1)$ and the surrounding isotropic fluid $(\phi=0)$. The term in $A_{\phi}$ results in phase ordering into two states with $\phi=1$ and $\phi=0$, and $K_{\phi}>0$ represents the surface tension that stabilises the interface between them. The term in $B$ slaves $S$ to $\phi$ so that the state with $\phi=1$ has $S=1$ and is nematic, whereas that with $\phi=0$ has $S=0$ and is isotropic. The last term in Eq. (2) assigns a free energy to distortions of the nematic order, assuming a single elastic constant $K_{\mathbf{Q}}$.

$\phi$ is a conserved variable which evolves in time according to the Cahn-Hilliard equation,

$$
\partial_{t} \phi+\nabla \cdot(\boldsymbol{u} \phi)=\Gamma_{\phi} \nabla^{2} \mu,
$$

where $\Gamma_{\phi}$ is a mobility and $\mu=\frac{\delta \mathcal{F}}{\delta \phi}$ is the chemical potential. The form of the left-hand side of Eq. (3) ensures that the colony responds to local flow fields.

We assume that the cells and surrounding fluid phase have the same viscosity and can be modelled as incompressible fluids of density $\rho$. The fluid velocity $\mathbf{u}$ obeys the equations

$$
\nabla \cdot \boldsymbol{u}=0, \quad \rho\left(\partial_{t}+\boldsymbol{u} \cdot \boldsymbol{\nabla}\right) \boldsymbol{u}=\boldsymbol{\nabla} \cdot \boldsymbol{\Pi},
$$

where $\boldsymbol{\Pi}$ is the stress tensor which includes the usual viscous stress, elastic stresses and a capillary term,

$$
\Pi^{\text {viscous }}=2 \eta \mathbf{E},
$$



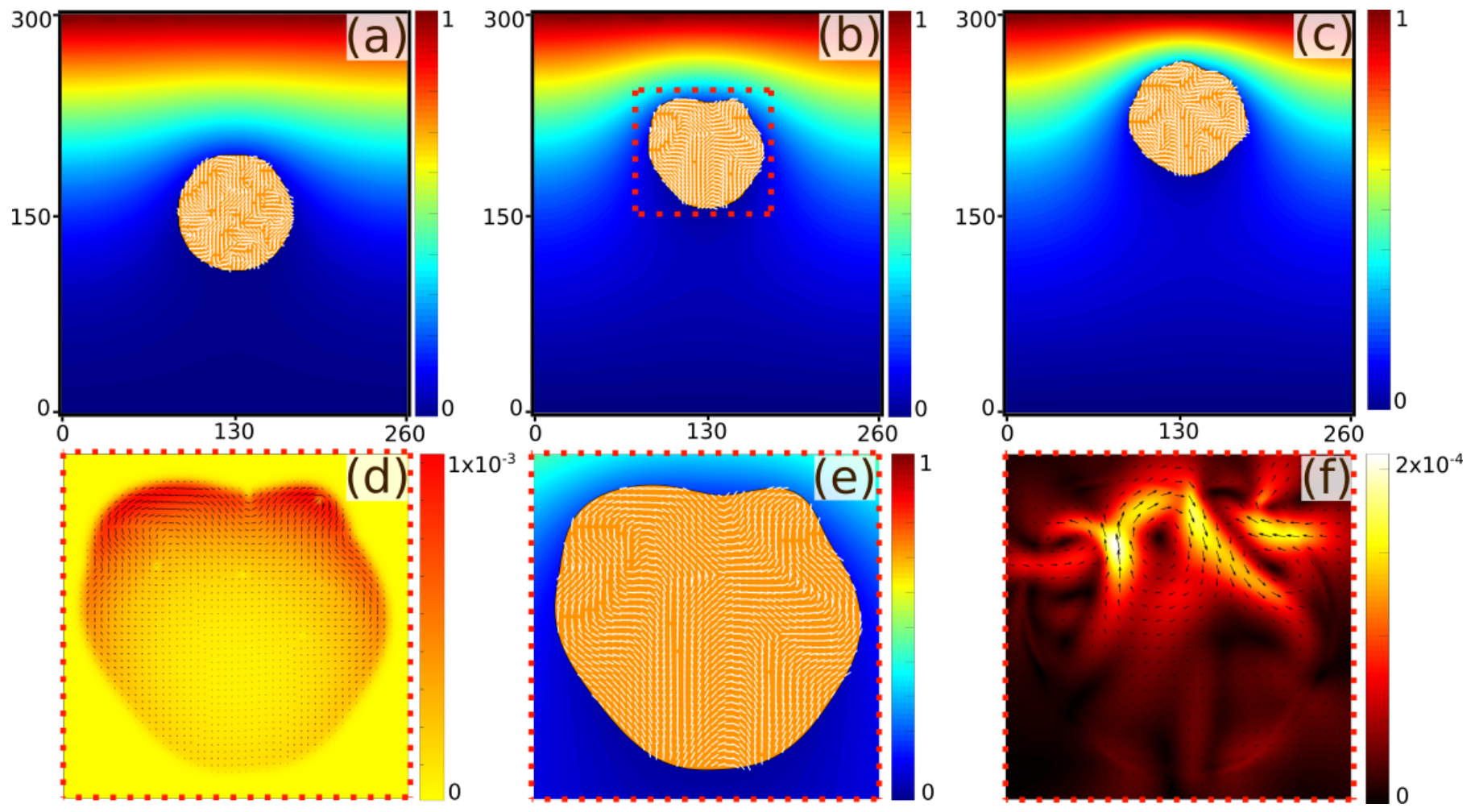

FIG. 1. Snapshots of the active colony at time-steps (a) 250,000 (b) 1,000,000 (c) 2,000,000. The colony is depicted in orange, with the corresponding director field $\mathbf{n}$ shown by the white dashes. The underlying colourmap represents the chemical concentration. (d)-(f) Magnified view of the section of the box outlined by red dashes in (b): (d) Coefficient of the active stress $\zeta C \phi S$. (e) Director field $\mathbf{n}$ inside the colony. (f) Magnitude of the velocity field, with the flow direction shown by the black arrows.

$$
\begin{gathered}
\boldsymbol{\Pi}^{\text {elastic }}=-P \mathbf{I}-\lambda \mathbf{H}+\mathbf{Q} \cdot \mathbf{H}-\mathbf{H} \cdot \mathbf{Q}-\boldsymbol{\nabla} \mathbf{Q} \frac{\delta \mathcal{F}}{\delta \boldsymbol{\nabla} \mathbf{Q}}, \\
\boldsymbol{\Pi}^{\text {cap }}=(\mathcal{F}-\mu \phi) \mathbf{I}-\nabla \phi\left(\frac{\delta \mathcal{F}}{\delta \nabla \phi}\right),
\end{gathered}
$$

respectively, where $\eta$ is the viscosity and $P=\rho / 3$ is the bulk pressure.

Of particular relevance here is the active contribution to the stress tensor,

$$
\mathbf{\Pi}^{\text {active }}=-\zeta C \phi \mathbf{Q},
$$

which is non-zero only within the cell colony. $\zeta$ is a measure of the strength of the activity. $\zeta>0$ and $\zeta<0$ correspond to an extensile or a contractile system respectively [16]. To couple the chemoattractant to the colony dynamics we assume that the active stress is proportional to the local concentration of the chemical field, $C$. In what follows we shall refer to $\zeta$, which is constant in space and time, as the activity and $\zeta C \phi S$, which varies within the colony and in time, as the active stress.

Lastly we write down a convection-diffusion equation for $C$ :

$$
\partial_{t} C+\boldsymbol{\nabla} \cdot(\boldsymbol{u} C)=\Gamma_{C} \nabla^{2} C-\alpha_{C} C \Theta(\phi-0.6),
$$

where $\Gamma_{C}$ is the diffusion coefficient of the chemical field. The final term in Eq. (9) describes depletion of the chemical at a rate controlled by $\alpha_{C}$. $\Theta(x)$ is a step function, equal to zero for $x \leq 0$ and unity for $x>0$ which ensures that depletion is restricted to within the cell layer.

The equations were solved on a lattice of size 260 in the $x$ direction by 300 in the $y$-direction (Fig. 1). A circular cluster of cells with $\phi=1$ was initialised near the centre of the simulation box. The components of $\mathbf{Q}$ were initialised randomly and $\mathbf{u}$ was set to zero. Neumann boundary conditions were chosen for the $\phi$-field and $\mathbf{Q}$ tensor, and free-slip boundary conditions were selected for the velocity field. However these are unimportant because the fluid at the walls is isotropic and passive and any velocities near the walls are insignificant. The chemical concentration field $\mathrm{C}$ was set to zero everywhere except along $y=300$ where it was set to 1 . It was allowed to diffuse out of the box at $y=1$, for all $x$ by setting the value of the field at the wall nodes such that the gradients at a wall node and its nearest neighbour were equal. Reflection boundary conditions were used for $\mathrm{C}$ at $x=0$ and $x=260$. The simulations were run for an initial 150,000 time steps after which the initialisation dynamics had relaxed, the colony had radius $r$, and the chemical field was in dynamic equilibrium. Data for the active colony was then collected for 2,000,000 timesteps.

We solved the equations of motion using a hybrid lattice Boltzmann method. Eqs. (1), (3) and (9) are solved using a predictor corrector method and Eq. (??) using a lattice Boltz- 

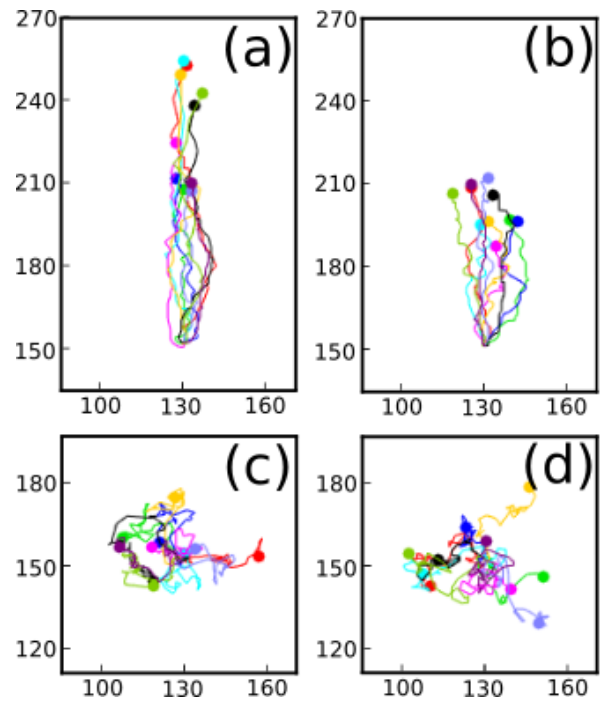

FIG. 2. Trajectories of the cluster centre-of-mass, for (a) $\zeta=0.008$ (b) $\zeta=-0.008$ in a chemical field gradient. (c) $\zeta=0.001$ (d) $\zeta=-0.001$ in a static homogenous chemical field. The values of $\zeta$ are adjusted in (c), (d) compared to (a), (b) to allow for the higher concentration of chemoattractant in the latter case.

mann algorithm $[21,22]$. The parameters used are $\Gamma_{Q}=0.3$, $\Gamma_{\phi}=0.2, \Gamma_{C}=0.15, A_{\phi}=0.1, B=0.001, K_{\phi}=0.1$, $K_{\mathbf{Q}}=0.0005, \alpha_{C}=0.005, \lambda=0.3, \rho=40, \eta=\frac{1}{6}$. The control parameters in the simulations are the cluster radius $r$ which takes values from 27 to 71 lattice sites and the activity $\zeta$ which varies from -0.02 to 0.02 .

Trajectories of Chemotactic Clusters: The dynamics of a cluster with radius $r=45$ and $\zeta=0.008$ is illustrated in Fig. 1. Panels (a), (b) and (c) correspond to snapshots at times $250,000,1,000,000$ and 2,000,000 respectively, with the underlying colourmap showing the chemical concentration field. The cell colony is clearly moving in the direction of increasing concentration. Figs. 1(d)-(f) enlarge the area outlined in red in panel (b). The active stress, $\zeta C \phi S$, is plotted in Fig. 1(d) indicating that it is only significant at the top of the cluster, differing by an order of magnitude between the top and bottom because of the adsorption of the chemoattractant by the cells. The director field $\mathbf{n}$ can be seen more clearly in subfigure (e). Finally, a snapshot of the magnitude and direction of the flow field is presented in Fig. 1(f). Note that the net motion towards the chemical source persists despite the apparent chaotic nature of the flows.

The trajectories of the centre-of-mass of the colony, for 10 different initial conditions, are compared in Fig. 2(a) (Fig. 2(b)) for an extensile (contractile) active nematic, with $\zeta=0.008(\zeta=-0.008)$. In all cases there is a net motion of the cells towards the chemical source, demonstrating that the colony is chemotactic for both extensile and contractile activity. For comparison we compare trajectories for the case where the chemical concentration is constant across the colony for extensile (Fig. 2(c)) and contractile (Fig. 2(d)) driving. Here the colony does not move in any preferred direction.
To quantify the efficiency of chemotaxis it is customary to use the chemotactic index, defined as the ratio of the displacement of the cluster centre-of-mass in the direction of the chemical source, $y$, to the total length of its trajectory. This varies from 0 for random motion to 1 for directed motion towards the chemical source. The chemotactic indices averaged over the trajectories in each frame of Fig. 2 are 0.84, 0.78, 0.0 and 0.03 for Figs. 2(a), (b), (c) and (d) respectively.

While the extensile and contractile colonies have similar chemotactic indexes, it is apparent from Fig. 2 that the extensile systems tend to move further than the contractile ones. The average rms velocity of the cluster centre-of-mass was $0.46 \times 10^{-4}$ for the extensile active clusters compared to $0.37 \times 10^{-4}$ for the contractile clusters (simulation units). This is a consequence of extensile active nematics generating stronger flows than contractile ones for the same value of the activity [23].

At this point, it is clear that the active colonies exhibit chemotaxis, but it is not obvious why this is the case. Next, we explain the physics underpinning this behaviour.

Mechanism: The director field of an extensile active nematic preferentially aligns tangentially to the boundary of a colony, whereas that of a contractile system tends to align radially [24]. This effect, which has been termed active anchoring, holds in the absence of any thermodynamic interface anchoring. Indeed radial cell polarization has been reported at the boundaries of cell colonies undergoing chemotaxis, suggesting that these systems can be described as contractile active nematics $[1,7,8]$.

The mechanism for active anchoring can be explained by considering the force density that arises from the active stress, $\nabla(-\zeta C \phi \mathbf{Q})$. Denoting the unit normal to the surface by $\mathbf{m}$, the force density is

$$
\mathbf{F}^{\text {active }}=\zeta\left\{\sigma\left((\mathbf{m} \cdot \mathbf{n}) \mathbf{n}-\frac{1}{2} \mathbf{m}\right)-S C \phi(\mathbf{n}(\nabla \cdot \mathbf{n})+(\mathbf{n} \cdot \nabla) \mathbf{n})\right\}
$$

where $\sigma=|\nabla S| \phi C+S|\nabla \phi| C-S \phi|\nabla C|$. Defining $\mathbf{l}$ as the unit-vector perpendicular to $\mathbf{m}$, the first term on the r.h.s. of Eq. (10), which dominates at the interface, leads to a force density acting along the colony surface $F_{\|}^{a c t i v e}=\zeta \sigma(\mathbf{m} \cdot \mathbf{n})(\mathbf{l} \cdot \mathbf{n})$. This force is zero only when the director $\mathbf{n}$ is normal or parallel to the surface. Otherwise it induces flows which realign n tangentially or radially for extensile or contractile clusters respectively [24].

Fig. 3(a)-(d) illustrates the active anchoring. Panel (a) is a snapshot showing that the director field of an extensile cluster tends to lie parallel to the surface. Panel (b) contrasts a contractile system where the director prefers to lie normal to the surface. To quantify the active anchoring Fig. 3(c) and (d) shows the corresponding normalised, time-averaged histogram of the angle between $\mathbf{n}$ and $\mathbf{m}$.

The surface anchoring propagates into the bulk because of elastic forces. This means that the distortions near the interface are preferentially bend-like in extensile systems and splay-like in contractile ones. In both cases this results in outwards radial flow (Fig. 3(e) and (f)). Since the concen- 


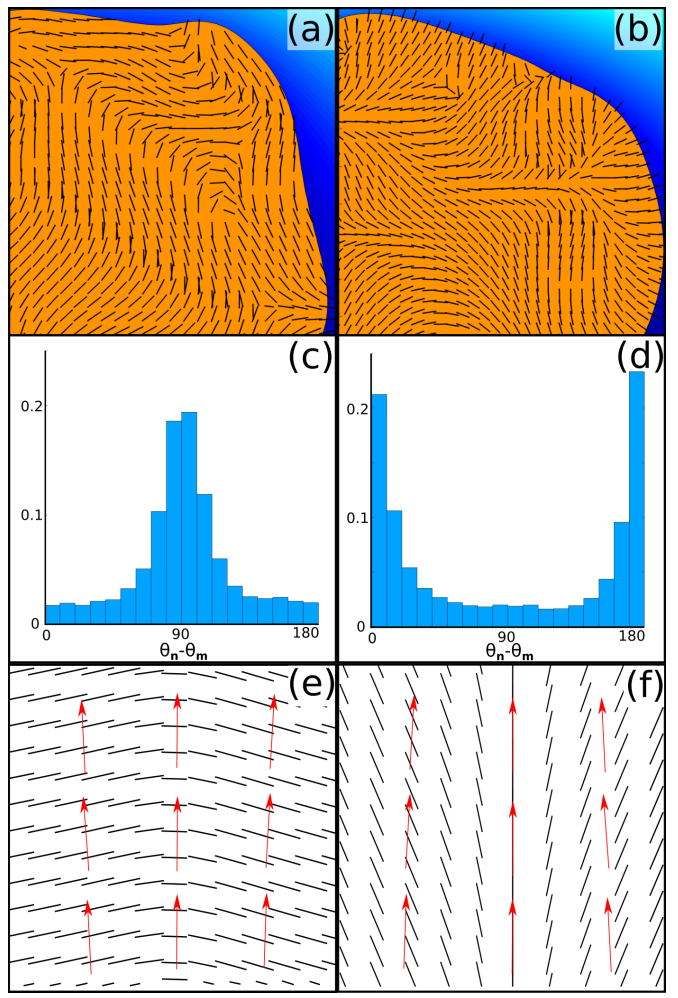

FIG. 3. Active alignment of the director at the surface of an active cluster: (a) extensile activity which results in parallel alignment, (b) contractile activity leading to normal alignment. Time-averaged, normalised histogram of the angle between the director and the surface normal, $\theta_{n}-\theta_{m}$ for (c) extensile activity, $\zeta=0.008$, (d) contractile activity, $\zeta=-0.008$. Schematic illustration of the flow field (red arrows) resulting from (e) a bend distortion in the director field of an extensile active nematic, (f) a splay distortion in the director field of a contractile active nematic.

tration of the chemoattractant is higher, and hence the active stress larger, in the part of the colony closest to the chemical source, the outward flows will be stronger there giving the imbalance that moves the colony up the chemical gradient. (These flows dominate those which arise from the $\sigma$-term in Eq. (10), which are directed inwards and so would act to make the cluster chemorepulsive.)

We next discuss quantitative results. Fig. 4(a) plots the rms velocity $V_{r m s}$ of the centre of mass of a cluster of radius $r=60$ against the activity for both contractile and extensile clusters. The cluster position is measured at intervals of $5 \times 10^{4}$ timesteps, and each data point is the result of averaging over ten independent simulations. Fig. 4(b) shows that the chemotactic index is approximately independent of the activity, and close to unity, indicating that the flows are well-aligned by the active anchoring.

Given we take a continuum approach our model is relevant to large, well connected clusters. Experiments using malignant lymphocyte cells [8] have shown that sufficiently large clusters chemotax with a velocity independent of their area in agreement with the dependence found here. However, the
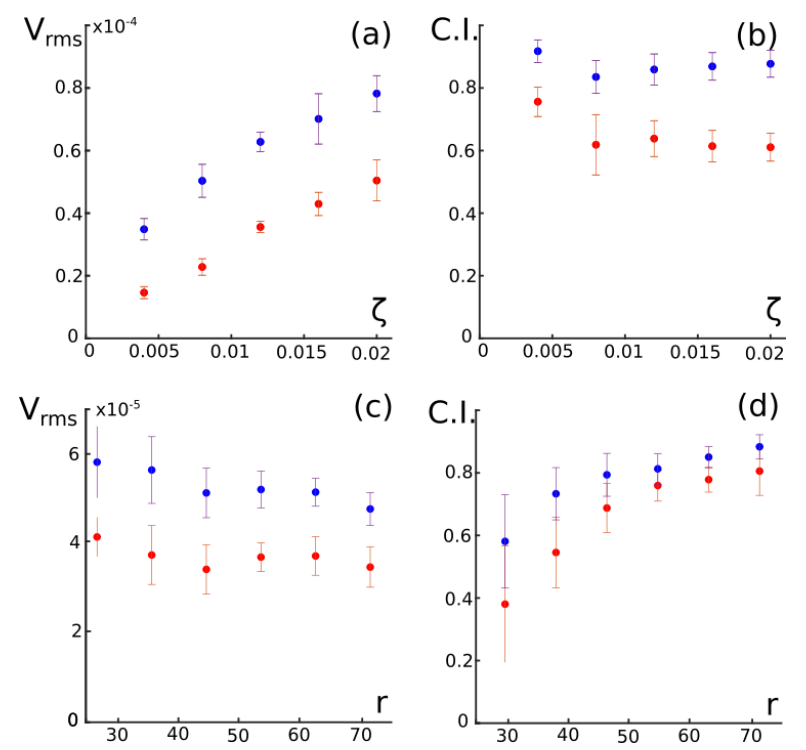

FIG. 4. (a) Root mean square velocity $V_{r m s}$ of the cluster centreof-mass as a function of activity, $\zeta$. (b) Chemotactic index, C.I., as a function of $\zeta$. (c) $V_{r m s}$ as a function of cluster radius $r$. (d) C.I. as a function of $r$. Blue (red) symbols denote extensile (contractile) activity.

experimental situation is far from clear. In [8] chemotaxis is seen only above a threshold size of 20 cells and in [1] neural crest cells are shown to chemotax even if they only have transient contact with their neighbours.

The chemotactic index, however, increases with cluster size but levels off as the cluster radius increases (Fig. 4(d)). This is because the flows in the smaller clusters show larger fluctuations. In particular the smallest clusters undergo obvious periods of rotation. This rotational motion can be explained by noting the tendency of the activity to drive flow vortices at a length scale commensurate with the size of these clusters [22, 25]. Similar behaviour has been observed for small clusters of lymphoid cells [8] allowing a mapping between simulation and physical parameters. In the experiments clusters with radius $\approx 30 \mu \mathrm{m}$ undergo periods of rotation for about 60 seconds. In the simulations the corresponding values are 40 lattice units and $10^{5}$ time steps. This maps the simulation value $V_{r m s} \approx 10^{-4}$ in lattice units on to $V_{r m s} \approx 10^{-1} \mu \mathrm{m} \mathrm{min}^{-1}$ in physical units which is in agreement with typical chemotactic velocities measured in experiments $[1,6,8]$.

Several mechanisms that can lead to collective chemotaxis have been proposed in the literature. These include chemical or mechanical interactions between cells initiated by a chemical gradient $[5,13]$, a coupling between contact inhibition of locomotion and a chemoattractant [12], and the existence of leader cells at the cluster rim that have a strong chemotactic response [8]. Here we show that interpreting a cell cluster as an active nematic leads naturally to collective chemotaxis. Active stresses give a net alignment of cells on the cluster surface, and the alignment propagates into the bulk resulting in stresses which drive flows directed outwards. If 
the strength of the activity depends on the local chemoattractant concentration, a chemical gradient results in unbalanced forces and hence motion up the gradient. Radial polarisation of cells at an interface has been observed in experiments, and the model explains this as arising from the contractile forces exerted by the cells $[1,2,6-8,26]$. Moreover the model reproduces the experimental observation that the net speed of chemotaxing clusters is independent of their size. This explanation of collective chemotaxis could be tested further by studying the shapes of cells in a chemotaxing cluster in more detail, by investigating the flow fields in the clusters, and by measuring the dependence of the chemotactic index and cluster speed on the magnitude of the active stress, which can be varied by changing the concentration of the chemoattractant.

[1] E. Theveneau, L. Marchant, S. Kuriyama, M. Gull, B. Moepps, M. Parsons, and R. Mayor, Dev. Cell 19, 39 (2010).

[2] R. Mayor and S. Etienne-Manneville, Nat. Rev. Mol. Cell Bio. 17, 97 (2016).

[3] B. A. Camley, Journal of Phys. Cond. Mat. 30, 223001 (2018).

[4] A. Shellard and R. Mayor, Journal of Cell Sci. 132 (2019).

[5] A. Gopinathan and N. S. Gov, Sem. in Cell and Dev. Bio. 93, 77 (2019).

[6] E. Camand, F. Peglion, N. Osmani, M. Sanson, and S. EtienneManneville, Journal of Cell Sci. 125, 844 (2012).

[7] A. Shamloo, Cytoskeleton 71, 501 (2014).

[8] G. Malet-Engra, W. Yu, A. Oldani, J. Rey-Barroso, N. S. Gov, G. Scita, and L. Dupr, Cur. Bio. 25, 242 (2015).

[9] A. Shellard, A. Szabó, X. Trepat, and R. Mayor, Science 362, 339 (2018).
[10] P. Friedl, J. Locker, E. Sahai, and J. E. Segall, Nat. Cell Bio. 14, 777 (2012).

[11] P. Van Liedekerke, M. M. Palm, N. Jagiella, and D. Drasdo, Comp. Par. Mech. 2, 401 (2015).

[12] B. A. Camley, J. Zimmermann, H. Levine, and W.-J. Rappel, Phys. Rev. Let. 116, 098101 (2016).

[13] B. A. Camley, J. Zimmermann, H. Levine, and W.-J. Rappel, PLoS Comput. Biol. 12, e1005008 (2016).

[14] B. A. Camley and W.-J. Rappel, Journal of Phys. D: App. Phys. 50, 113002 (2017).

[15] J. Prost, F. Jülicher, and J.-F. Joanny, Nat. Phys. 11, 111 (2015).

[16] A. Doostmohammadi, J. Ignés-Mullol, J. M. Yeomans, and F. Sagués, Nat. Com. 9, 3246 (2018).

[17] K. Kawaguchi, R. Kageyama, and M. Sano, Nature 545, 327 (2017).

[18] T. B. Saw, A. Doostmohammadi, V. Nier, L. Kocgozlu, S. Thampi, Y. Toyama, P. Marcq, C. T. Lim, J. M. Yeomans, and B. Ladoux, Nature 544, 212 (2017).

[19] C. Blanch-Mercader, V. Yashunsky, S. Garcia, G. Duclos, L. Giomi, and P. Silberzan, Phys. Rev. Lett. 120, 208101 (2018).

[20] G. Duclos, C. Blanch-Mercader, V. Yashunsky, G. Salbreux, J.F. Joanny, J. Prost, and P. Silberzan, Nat. Phys. 14, 728 (2018).

[21] T. Krüger, H. Kusumaatmaja, A. Kuzmin, O. Shardt, G. Silva, and E. M. Viggen, The Lattice Boltzmann Method (Springer, Cham, 2016).

[22] J. Hardoüin, R. Hughes, A. Doostmohammadi, J. Laurent, T. Lopez-Leon, J. M. Yeomans, J. Ignés-Mullol, and F. Sagués, Comm. Phys. 2, 121 (2019).

[23] S. P. Thampi, R. Golestanian, and J. M. Yeomans, Phil. Trans. Series A, Math., Phys., and Eng. Sci. 372, 20130366 (2014).

[24] M. L. Blow, S. P. Thampi, and J. M. Yeomans, Phys. Rev. Lett. 113, 248303 (2014).

[25] T. N. Shendruk, A. Doostmohammadi, K. Thijssen, and J. M. Yeomans, Soft Matter 13, 3853 (2017).

[26] O. Ilina and P. Friedl, Journal of Cell Sci. 122, 3203 (2009). 